\title{
Aplicativos para o ensino e aprendizagem de Química
}

\section{Aline Grunewald Nichele, IFRS - Campus Porto Alegre, aline.nichele@poa.ifrs.edu.br} Eliane Schlemmer, UNISINOS, elianes@unisinos.br

Resumo. A distribuição de dispositivos móveis, como tablets, para professores de ensino médio das escolas públicas, viabiliza o desenvolvimento do mobile learning, impulsionando mudanças na forma como os sujeitos se relacionam com a informação e produzem o conhecimento. Adicionalmente, na área da Educação Química, vislumbrase que os tablets e aplicativos podem proporcionar situações de aprendizagem que eram restritas, por exemplo, a softwares caros. Nesse contexto e com o objetivo de conhecer os aplicativos para a Educação Química e o crescimento de sua oferta para incentivar sua adoção na educação, trazemos, nesse artigo, os resultados de uma pesquisa sobre aplicativos para tablet relacionados à área, realizada de maio/2012 a maio/2014, apresentando a evolução do número de aplicativos, os temas mais recorrentes e finalizamos indicando aplicativos para a Educação Química.

Palavras-chave: dispositivos móveis, aplicativos, tablet.

Abstract. The distribution of mobile devices like tablets to high school teachers of public Brazilian schools, enable the development of mobile learning in the educational context, changing the way people face information and handle knowledge. In Chemistry Education area, tablets and Apps provide learning experiences and situations which used to be limited, in the past. Facing the opportunities of the wide range and low prices of Apps for tablets as well as aiming potencial applications in Chemistry Education, we would like to encourage the adoption of tablets and Apps in teaching and learning. Therefore, we researched the evolution of the Apps amounts, from May/2012 to May/2014, and the most common and recurrent subjects in Chemistry Education. Finally, we suggested some useful Apps, based on the results of our research.

Keywords: mobile devices, Apps, tablet

\section{INTRODUÇÃO}

Os dispositivos móveis com conexão sem fio e interface sensível ao toque (touch-screen), tais como tablets e smartphones, associados a diferentes aplicativos têm proporcionado mudanças na forma de nos relacionarmos com a informação e produzir conhecimento, apresentando significativo potencial para transformar a maneira de ensinar e de aprender. Elas proporcionam aos professores e estudantes mobilidade e interface fácil de usar, podendo assim, contribuir para implementar diferentes estratégias de ensino e de aprendizagem, ampliando as possibilidades de ação e interação entre sujeitos, sujeitos e meio (incluindo os próprios dispositivos, aplicativos e o ambiente - local geográfico onde os sujeitos se encontram), bem como os processos de colaboração e a cooperação.

A disseminação desse tipo de dispositivo tem impulsionado o desenvolvimento de novos aplicativos (Apps) com potencial para incrementar o aprendizado dentro e fora da sala de aula. Há diversos tipos de Apps disponíveis, tais como, jogos, mídias sociais, livros (entre eles dicionários, enciclopédias), revistas, bem como aplicativos específicos para educação, para navegação na internet, para gerenciamento e organização de atividades e processos. 
No contexto escolar, a flexibilidade espacial e temporal propiciada pelos dispositivos móveis com conexão sem fio, como os tablets, conferem novas possibilidades na educação, como o prolongamento das atividades escolares para além dos limites físicos da escola, além de descentralizá-la no que se refere ao docente como única fonte de informação. Essas características ampliam, entre outras possibilidades, o desenvolvimento do mobile learning, que é uma modalidade educacional em que processos de aprendizagem ocorrem com o uso de dispositivos móveis, conectados a redes de comunicação sem fio, e tem como característica fundamental a mobilidade dos aprendizes, que podem estar distantes uns dos outros e também dos espaços formais de educação. Essa mobilidade, além de física e temporal, é também tecnológica, conceitual e sociointeracional (Saccol, Schlemmer e Barbosa, 2011).

Além dessas possibilidades e características, os tablets podem se destacar nos processos de ensino e aprendizagem de Química pelo potencial de proporcionar melhores simulações e modelos, permitindo a visualização e manipulação digital virtual da representação de estruturas químicas por meio de telas touch-screen, o acesso a tabelas de dados químicos, entre outras possibilidades.

Entretanto, um dos requisitos fundamentais para implementar estratégias de ensino e de aprendizagem utilizando dispositivos móveis com conexão sem fio nas escolas é uma infraestrutura tecnológica adequada, a qual inclui acesso a internet e dispositivos móveis como os tablets.

No Brasil, no que se refere à infraestrutura, algumas políticas públicas do governo federal vêm sendo implementadas por meio de projetos e programas, tais como o "Programa Banda Larga nas Escolas", o "Programa Computador Portátil para Professores", o "Programa Nacional de Informática na Educação - ProInfo", o "Projeto Um Computador Por Aluno - UCA”, para inserção das tecnologias digitais na educação. Recentemente, no ano de 2012, foi apresentado o projeto Educação Digital Política para computadores interativos e tablets para as escolas de ensino médio, que prevê, entre outras ações, a entrega de tablets $^{1}$ aos professores das escolas públicas, que iniciou em 2013.

Esse projeto, nos instigou a investigar os aplicativos disponíveis para tablet que podem contribuir para a implementação e consolidação de estratégias de ensino e de aprendizagem de Química no contexto da mobilidade (mobile learning) e da possibilidade de se ampliar o nível de compreensão da Química por meio de simulações, modelos, exercícios, jogos e acesso a tabelas de dados disponíveis em lojas virtuais.

Nesse sentido, entendemos que nos processos de ensino e de aprendizagem de Química, a adoção de dispositivos móveis - tais como os tablets - e de aplicativos vinculados a Química, pode viabilizar oportunidades não possíveis em salas de aula convencionais e em laboratórios presenciais físicos, incluindo a possibilidade de superação de um dos desafios da Educação Química, que é proporcionar ao estudante correlacionar um fenômeno em sua dimensão macroscópica com as dimensões submicroscópica e simbólica (Giordan, 2008). Entretanto, na literatura há poucos estudos envolvendo tablets, bem como smartphones e mobile learning, para o ensino e aprendizagem de Química (Libman e Huang, 2013; Ekins et al., 2013; Feldt et al., 2012; Bonifacio, 2012; Williams e Pence, 2011; García-Ruiz et al., 2012). Assim, no momento em que os professores das escolas públicas brasileiras de nível médio estão recebendo tablets, temos a preocupação de ampliar a possiblidade de adoção desses dispositivos nos processos de ensino e de aprendizagem de Química e a encorajar os professores da área a utilizá-los. A etapa inicial dessa pesquisa, apresentada nesse artigo, busca pesquisar os aplicativos para tablets com potencial para incrementar a 
prática docente. Dessa maneira, nesse trabalho temos como objetivo investigar e verificar a evolução da disponibilidade de aplicativos para tablet com potencial para os processos de ensino e aprendizagem de Química, bem como identificar os temas dessa área do conhecimento mais recorrentes nos Apps e sugerir alguns aplicativos aos docentes dessa área da ciência.

\section{APLICATIVOS PARA TABLETS PARA O ENSINO E APRENDIZAGEM DE QUÍMICA}

Com o intuito de conhecer os aplicativos para tablets com potencial para ser utilizado na Educação Química foram consideradas e testadas as lojas virtuais "Play Store" (para o sistema operacional Android, característico da maioria das marcas de dispositivos móveis, incluindo o "Positivo" e o "CCE", tablets que estão sendo distribuídos aos professores de ensino médio de escolas públicas brasileiras) e a "App Store” (para o sistema operacional iOS, característico dos produtos da Apple).

As pesquisas efetuadas na "Play Store" foram realizadas por meio de um tablet educacional da marca Positivo (distribuído pelo Ministério da Educação). Na "Play Store” há a opção de pesquisa por "Apps”, “Jogos”, "Filmes”, "Livros"; e a possibilidade de se inserir palavras de busca. Para o desenvolvimento dessa pesquisa utilizamos a opção “Apps”, para a qual é possível utilizar a seleção de categorias específicas, incluindo a categoria “educação”, mas nenhuma delas foi selecionada com o intuito de se contemplar todos os Apps com potencial para a Educação Química, mesmo que não estejam associados especificamente a essa categoria.

As pesquisas por Apps na "App Store" foram efetuadas por meio de aplicativo específico (App Store), utilizando-se um iPad 2 como dispositivo móvel. Na “App Store” é possível utilizar-se como filtros da pesquisa a seleção de categorias específicas, entretanto, mesmo que exista a categoria “educação” essa também não foi selecionada, pois observamos que alguns aplicativos que podem ser úteis para a Educação Química pertencem a outras categorias.

Para alcançar os objetivos dessa pesquisa, a “App Store” apresentou melhor estrutura para a busca de aplicativos, tanto no que se refere à combinação de critérios de pesquisa, bem como de informar o número de aplicativos que retornam a uma busca, ao contrário da "Play Store", que sempre retorna o mesmo número de aplicativos, ou seja, não considera todos os disponíveis e/ou insere aplicativos relacionados, mas não específicos à pesquisa realizada. Mesmo se tratando de diferentes lojas virtuais, para diferentes sistemas operacionais, há Apps que são comuns a ambas.

Assim, a investigação quanto ao aspecto quantitativo de aplicativos disponíveis com potencial para o ensino e aprendizagem de Química foi realizada na "App Store”. O conhecimento do número de aplicativos na loja virtual nos permite presumir o quão evoluído está o desenvolvimento de Apps para a área de Química, de forma a viabilizar a utilização desses para fins educacionais, estando esse pressuposto presente na sua criação ou não.

Com relação ao tipo de “dispositivo" foram registradas as quantidades de aplicativos disponíveis para tablet (iPad); bem como foram registrados os números de aplicativos totais em relação ao custo para download, discernindo-se a quantidade de Apps gratuitos para download. 


\subsection{Aplicativos para tablet: número de Apps disponíveis}

A simplicidade e rapidez para utilização, a interface amigável, a facilidade para instalar os aplicativos que aos tablets dão múltiplas funcionalidades, são aspectos que contribuem para a sua rápida aceitação e disseminação mundial. Dadas essas características, pensar a utilização desses dispositivos no contexto educacional torna-se natural. Entretanto, a perspectiva educacional é propiciada por meio da instalação de aplicativos específicos, que conferem aos tablets a possibilidade de se desenvolver atividades no âmbito dos processos de ensino e de aprendizagem. Com o intuito de identificar os aplicativos para tablets com potencial para os processos de ensino e aprendizagem em Química, mensalmente, no interstício de 25 meses, de maio/2012 a maio/2014, efetuamos a busca de Apps para o iPad, na loja virtual “App Store”. Para identificá-los foi utilizada a palavra "chemistry" como palavra de busca. O total de aplicativos para iPad encontrados está sumarizado na Figura 1.

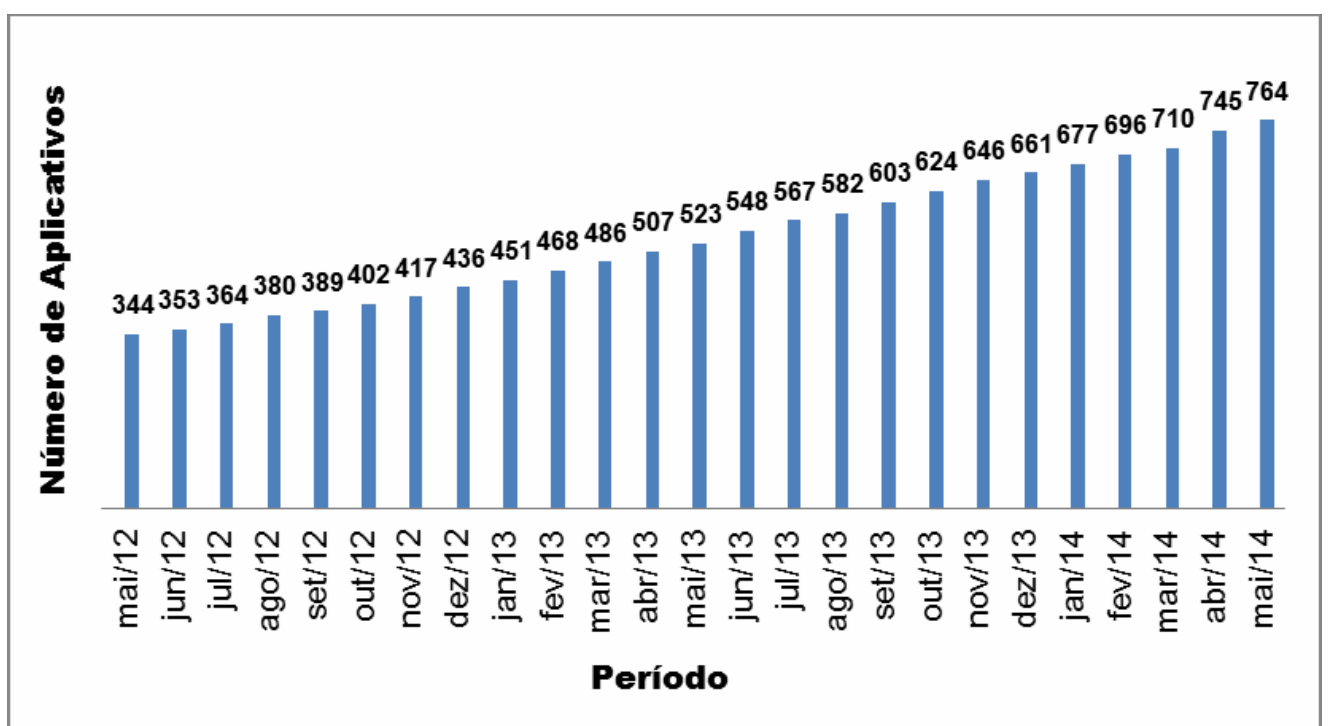

Figura 1 - Gráfico da evolução mensal do número total de aplicativos para iPad disponíveis na “App Store” para a Educação Química.

Nesse interstício de maio/2012 a maio/2014, o número de aplicativos para iPad teve um crescimento de cerca de $122 \%$, de 344 para 764 aplicativos, indicando a disseminação mundial desse tipo de tecnologia no contexto dos processos de ensino e aprendizagem da área de Química.

A adoção de tablets e aplicativos no contexto educacional em países em desenvolvimento como o Brasil, mesmo que programas governamentais viabilizem o acesso da população a esses dispositivos, pode ser prejudicado pelo custo de download de alguns Apps, ainda que este seja da ordem de poucos reais. Assim, discernimos nas buscas por aplicativos na "App Store" os aplicativos gratuitos para download, pois entendemos esta característica como um facilitador para sua inserção na escola. O total de aplicativos gratuitos para iPad no interstício de maio/2012 a maio/2014 pela palavra “chemistry” está sumarizado na Figura 2. 


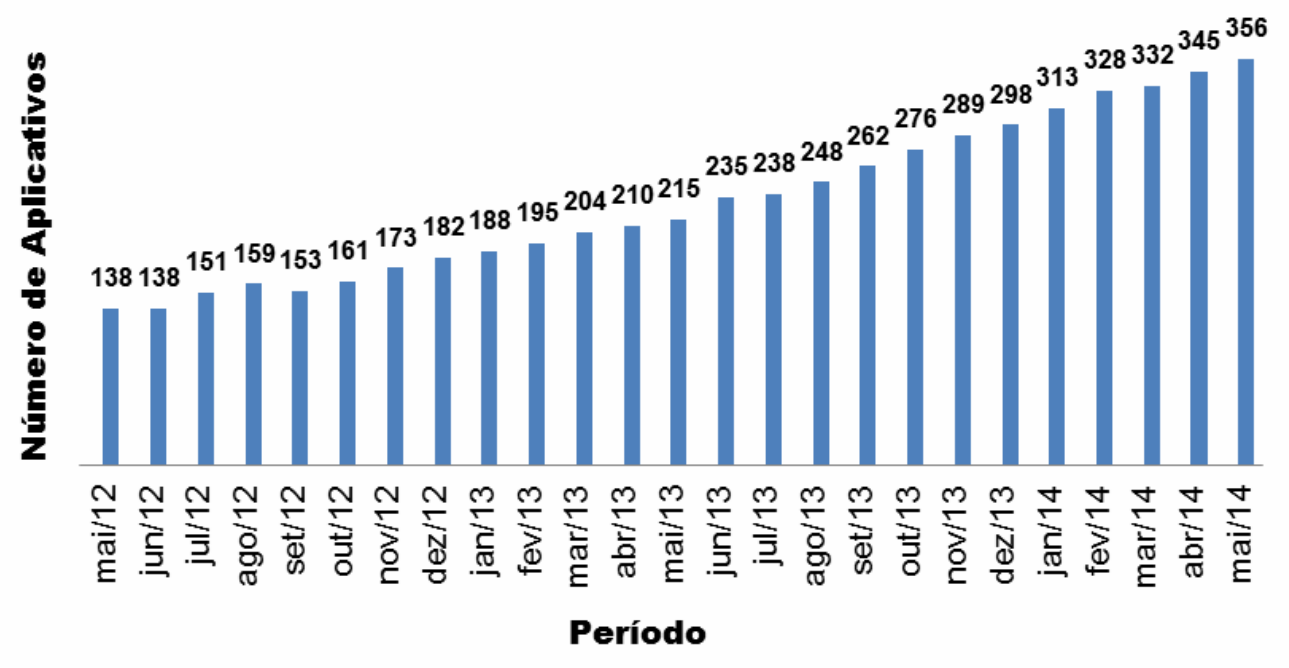

Figura 2 - Gráfico da evolução mensal do número de aplicativos para iPad gratuitos para download disponíveis na “App Store”.

Um importante crescimento do número de aplicativos sem custo para download de cerca de $158 \%$ foi observado no período analisado. Em números absolutos este incremento foi de 138 Apps gratuitos para iPad em maio/2012 para 356 em maio/2014.

Porém, a comparação entre o número total de aplicativos para iPad com potencial para a Educação Química (Figura 1) e o de Apps gratuitos (Figura 2) explicitam que a quantidade desses é significativamente inferior. Considerando-se os dados referentes a maio/2014, eram ao total 764 para 356 aplicativos sem custo para download. Ainda assim, consideramos que o número de Apps gratuitos para iPad é significativo, compondo um universo de boas possibilidades para o uso na educação química.

\subsection{Aplicativos para tablet: temas da Química abordados}

Mais importante que identificar a quantidade de aplicativos para tablets que estão disponíveis para os processos de ensino e aprendizagem em Química, é identificar quais temas desta área do conhecimento são contemplados nos Apps e, portanto, possíveis de serem utilizados pelos professores e alunos nos processos de ensino e de aprendizagem.

A análise do total de aplicativos disponíveis na “App Store” em maio/2014 permitiu verificar que o tema da Química mais recorrente nesses Apps é tabela periódica; seguido aspectos relacionados a ligações químicas, estrutura e modelo molecular, e, química orgânica, desde a identificação de funções orgânicas até mecanismos de reações. Na Figura 3 é possível observar o número de aplicativos totais e gratuitos para o iPad disponíveis para os temas mais recorrentes nos Apps. 


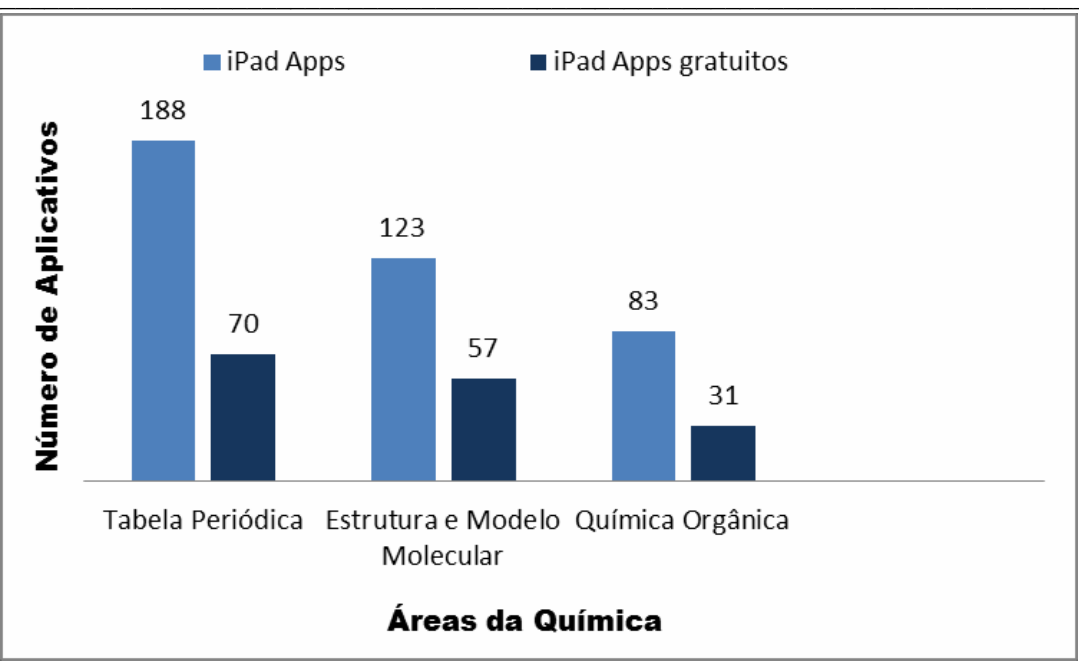

Figura 3 - Gráfico do número de aplicativos para iPad por temas da Química, disponíveis na “App Store” em maio/2014.

Da mesma maneira que se verifica mês a mês uma crescente disponibilidade de Apps relacionados a temas da Química para o sistema operacional iOS, também é crescente a disponibilidade de Apps para o sistema operacional Android, embora sua quantificação não seja obtida de maneira direta na loja virtual. Em maio/2014, por exemplo, havia disponível na "Play Store" 125 Apps relacionados à tabela periódica.

Verificamos que muitos dos aplicativos encontrados nessa pesquisa estão disponíveis apenas para um sistema operacional (Android ou iOS), entretanto, buscando encorajar os docentes da área de Educação Química a utilizá-los nos processos de ensino e de aprendizagem, independente do tablet e do sistema operacional que utilizam, selecionamos alguns Apps comuns a ambos sistemas operacionais, todos eles gratuitos. Para apresentar os ícones representativos de cada um dos Apps sugeridos utilizamos a imagem da tela do tablet educacional da Positivo (Figura 4), um dos modelos que hoje é distribuído aos professores das escolas públicas de nível médio brasileiras. 


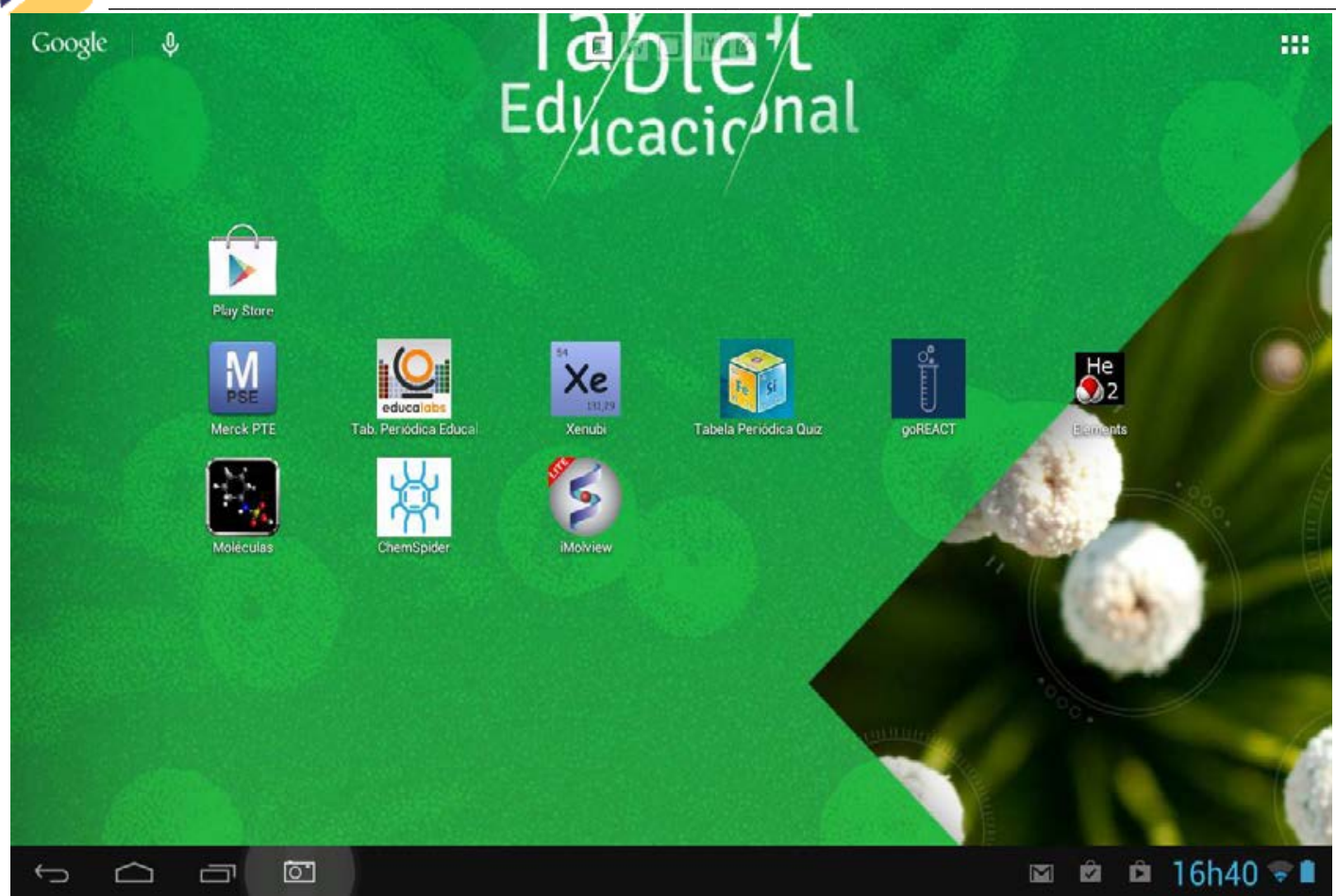

Figura 4 - Tela do tablet educacional Positivo com alguns Apps sugeridos.

Na Figura 4 os ícones dos Apps foram organizados em três linhas. Na primeira está o ícone de acesso à "Play Store”, loja virtual por meio da qual esses, e muitos outros, Apps podem ser instalados no tablet educacional, bem como em outros dispositivos que utilizem o sistema operacional Android.

Na segunda linha estão os Apps que sugerimos relacionados à tabela periódica, embora existam muitos outros. Nominalmente, eles são, respectivamente, da esquerda para a direita: "Merck PTE HD”, “Tabela Periódica Educalabs”, "Xenubi - Tabela Periódica”, “Tabela Periódica Quiz”, “goREACT” e "Elements - Periodic Table”.

Entre esses Apps, os que possuem conteúdo em língua portuguesa são: “Tabela Periódica Educalabs”, “Xenubi - Tabela Periódica” e “Tabela Periódica Quiz”.

“Tabela Periódica Educalabs” é uma tabela periódica interativa 3D. Proporciona a personalização da disposição dos elementos (tabela padrão ou estendida) e possibilita a visualização da representação do átomo com seus níveis de energia e respectivos elétrons, bem como algumas características gerais de cada um dos elementos, dados de propriedades periódicas, dados de propriedades físicas, informações históricas dos elementos (tais como descoberta e isolamento do elemento).

"Xenubi - Tabela Periódica” e "Tabela Periódica Quiz" são exercícios apresentados na forma de jogos. Em "Xenubi - Tabela Periódica” o usuário é desafiado a relacionar e comparar a posição de elementos com suas propriedades periódicas, e em “Tabela Periódica Quiz” a relacionar nomes de elementos a seus respectivos símbolos.

“Merck PTE HD”, "goREACT” e "Elements - Periodic Table” têm conteúdo em língua inglesa e tratam-se de Apps que essencialmente fornecem uma tabela periódica para consulta de dados.

Na terceira linha estão os Apps que sugerimos relacionados a estruturas químicas. Nominalmente, eles são, respectivamente, da esquerda para a direita: "Moléculas", "ChemSpider Mobile” e "iMolview Lite".

O App "Moléculas” tem conteúdo em língua portuguesa e, com ele é possível rotacionar, mover e dar zoom para várias moléculas (cerca de 270) que estão disponíveis 
na biblioteca do aplicativo. A busca por essas moléculas pode ser realizada pelo nome ou pela fórmula molecular.

“ChemSpider Mobile” e “iMolview Lite” têm conteúdo em língua inglesa. O "ChemSpider Mobile” permite visualizar a representação de moléculas e suas estruturas bidimensionais de sua biblioteca e, a partir do App, pode-se acessar o site da ChemSpider e visualizar dados e maiores informações acerca da molécula visualizada. Além disso, a partir da representação da estrutura da molécula no App "ChemSpider" pode-se, na versão disponível na "Play Store”, editá-la no App; e, na versão disponível na "App Store" é possível visualizá-la tridimensionalmente em outro App, por exemplo, no App "iMolview Lite”, e "manipulada" (rotacionada, ter seu tamanho aumentado ou diminuído).

Cabe lembrarmos que mais importante que os dispositivos móveis e aplicativos disponíveis, é o uso inteligente dessa tecnologia, em especial na busca da superação da reprodução de modelos de aprendizagem fundamentados na entrega de conteúdos, como a denominada por Freire (1987) de "educação bancária”. Nesse sentido, a utilização de dispositivos móveis e aplicativos no contexto educacional deve ser planejada para ir além de uma mera transposição de conteúdo do meio analógico para o digital, ou restringida ao uso das tecnologias digitais para a busca de informação, não atingindo a expectativa de produção de conhecimento e de desenvolvimento da autonomia do estudante. No planejamento docente, deve-se considerar a adoção dessas tecnologias articulada a metodologias problematizadoras, com uma mediação pedagógica aberta e flexível para atender as demandas oriundas da educação suportada pelas tecnologias digitais, em que a aprendizagem pode ocorrer individualmente, bem como pela interação e colaboração em grupos.

\section{CONSIDERAÇÕES FINAIS}

A distribuição de tablets aos professores das escolas públicas de ensino médio brasileiras, aliada à evolução do número de aplicativos, destaca o potencial desse dispositivo como uma real tendência, capaz de provocar inovação nos processos de ensino e de aprendizagem de Química, por meio de seus aplicativos e de características inerentes a esse tipo de dispositivo, como a interatividade e a mobilidade, desde que vinculados a transformações nas metodologias, práticas e processos de mediação pedagógica. Acreditamos que o conhecimento e a adoção de dispositivos móveis com conexão sem fio, aliada ao crescente número de aplicativos com potencial para a área de Educação Química, pode mobilizar os professores da área, em especial os que receberam os tablets, a desenvolver atividades no contexto da Educação Química. Entretanto, entendemos que, somente o conhecimento dos aplicativos para tablets, não é suficiente para que ocorra inovação na Educação Química, pois para que a inovação aconteça é necessário, primeiro, que os professores atribuam sentidos, signifiquem o uso, no que se refere ao seu próprio processo de aprendizagem, estando esse processo fundamentado numa perspectiva mais ampla, que envolve antes, uma clareza epistemológica no que se refere à compreensão de como os sujeitos aprendem na interação com esses dispositivos e aplicativos, na relação com as especificidades da área do conhecimento (Química), para que dessa forma, possam desenvolver metodologias, práticas e processos de mediação pedagógica capazes de provocar mudanças significativas na forma de se ensinar e de aprender, considerando as especificidades desses dispositivos, bem como o contexto da mobilidade. 


\section{REFERÊNCIAS BIBLIOGRÁFICAS}

BONIFÁCIO, V. D. B. QR-Coded Audio Periodic Table of the Elements: A MobileLearning Tool. Journal of Chemical Education. n. 89, p. 552-554, 2012.

EKINS, S.; CLARK, A. M.; WILLIAMS, A. J. Incorporating green chemistry concepts into mobile chemistry applications and their potential uses. ACS Sustainable Chemistry \& Engineering, v.1, n.1, p. 8-13, 2013.

FELDT, J.; MATA, R. A.; DIETERICH, J. M. Atomdroid: A Computational Chemistry Tool for Mobile Platforms. Journal of Chemical Information and Modeling, v.52, p. 1072-1078, 2012.

FREIRE, P. Pedagogia do Oprimido. 17. ed. Rio de Janeiro: Paz e Terra, 1987.

GARCÍA-RUIZ, M. A.; VALDEZ-VELAZQUEZ, L. L.; GÓMEZ-SANDOVAL, Z.

Estudio de usabilidade de visualización molecular educativa em um telefono inteligente. Química Nova, v.35, n.3, p. 648-653, 2012.

GIORDAN, M. Computadores e linguagens nas aulas de ciências: uma perspectiva sociocultural para compreender a construção de significados. Ijuí: Editora Unijuí, 2008.

LIBMAN, D.; HUANG, L. Chemistry on the Go: Review of Chemistry Apps on Smartphones. Journal of Chemical Education, n. 90, p. 320-325, 2013.

SACCOL, A.; SCHLEMMER, E.; BARBOSA, J. M-learning e u-learning: novas perspectivas das aprendizagens móvel e ubíqua. São Paulo: Pearson Prentice Hall, 2011. WILLIAMS, A. J.; PENCE, H. E. Smart Phones, a Powerful Tool in the Chemistry Classroom. Journal of Chemical Education, n.88, p. 683-686, 2011.

\footnotetext{
${ }^{1}$ Fabricados no Brasil pelas empresas CCE Digibras e Positivo Informática, com respectivamente 7 e 10 polegadas, a tela do equipamento possui resolução de 1024x600 pixels, armazenamento interno de 16 gigabytes e processador de um giga-hertz, funcionam com sistema operacional Android. O aparelho conecta-se a redes sem fio (tecnologia $\mathrm{Wi}-\mathrm{Fi}$ ).
} 